Stefano Monti*

\title{
REDUCTION OF THE LOCAL STRESS FIELD AROUND HOLES THROUGH POROUS SHAPED STRUCTURES
}

Geometrical discontinuities in mechanical components are detrimental for the mechanical properties of the product itself. Actually, in proximity of such features, the stress increases due to the stress concentration factor, that in the case of a circular hole is equal to 3. Several solutions have been proposed to reduce the stress concentration value. In the present article, the application of a particular porous pattern that can be obtained by laser cutting with the appropriate finishing requirements is introduced in order to modify the local stress field and reduce the stress concentration value near the hole boundary.

Keywords: void pattern, stress concentration factor, stress field

\section{Introduction}

Holes are crucial features in mechanical components due to the stress concentration factor arising in the proximity of hole boundary. However, details with holes are widely used in engineering to guarantee some function, such as bolt or rivet joints. Anyway, the local stress concentration introduced by holes strongly limits the fatigue strength and is still a subject able to attract the interest of researchers and engineers. Through the years, many studies were proposed aimed at estimating the stress concentration factor both for isotropic [1], [2], [3] and orthotropic plates [4], [5], [6], [7]. Holed components are interesting even today, due to the increased manufacturing and machining possibilities offered by the new technologies that allow to define and put in practice original solutions for the local stress reduction. Meguid [8] introduced the so-called defense hole system in a uniaxially loaded plate with a reduction of $7.5-11 \%$ of the stress concentration factor. In addition, other authors employed the defense hole systems for reducing the stress concentration factor [9], [10]. Jindal [11] investigated the influence of auxiliary holes on the static stress concentration around a central hole numerically by the finite element method (FEM) and experimentally by a two-dimensional photo-elastic method. Providakis et al. [12] presented a parametric study for the reduction of the dynamic stress concentration around a circular hole in an elastic finite plate subjected to a suddenly applied loading acting in its plane by using the BEM.

Alternatively, shaped porous structures have been recently studied and applied to metal sheet in order to achieve a specific material behavior, e.g. auxetic behavior, and manufactured by using additive manufacturing techniques. Grima et al. [13] found that conventional materials containing diamond or star shaped holes, arranged in a periodic structure, exhibits various Poisson's ratio values that can reach even negative values (auxetic behavior). Such evidence was found by Taylor et al. [14] by applying a pattern of elliptical voids, investigating different aspect ratios. As a consequence of the cited studies, Javid et al. [15] moved their interest to the stress behavior rather than on the material deformation and proposed a method for enhancing the fatigue life of turbine blades by replacing the commonly used circular holes with a novel orthogonal S-shaped void pattern, realized by means of the laser drilling. Such a novel pores design induces a soft mode of deformation based on rotation of the material around said voids, redistributing the stress field and modifying even the crack propagation phenomenon within the structure. The literature lacks in studies about employing the void patter for enhancing component's mechanical performances. As a consequence, there is a wide range of fields in which the void patterns can be employed for mechanical performance improvement.

The present study considers the case of a circular hole in an infinite plate under remote uniaxial load and proposes a C-shaped void pattern to redistribute the local stress field around the hole; in such a way the stress concentration factor can be consequently reduced. Moreover, the shape hereafter introduced is designed to be realized by means of the laser cutting technology. Furthermore, the introduced pattern is defined passing through the following steps:

- Introduction of the analytical description of the case study in order to comprehend which is the critical region;

- In defining the said pattern, it is necessary to define a suitable feature shape that is able to reduce the induced stress concentration factor as much as possible;

- Then, the distance between the two contiguous pattern rows is defined as the one able to better redistribute the stress field in the critical region;

- The same as before is done for defining the most suitable distribution of the features, considering a three-row pattern.

\footnotetext{
* Stefano Monti

Department of Mechanical Engineering, Politecnico di Milano, Italy

E-mail: stefano.monti@polimi.it
} 
a)

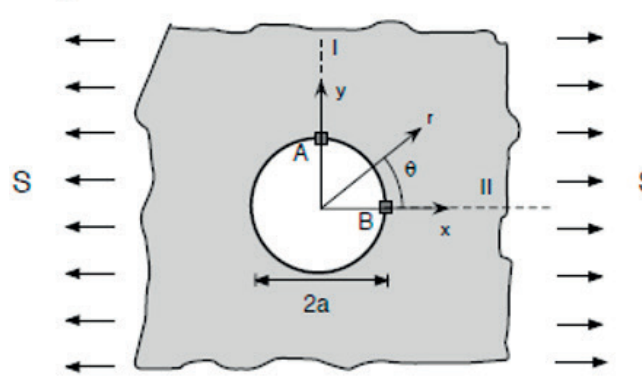

b)

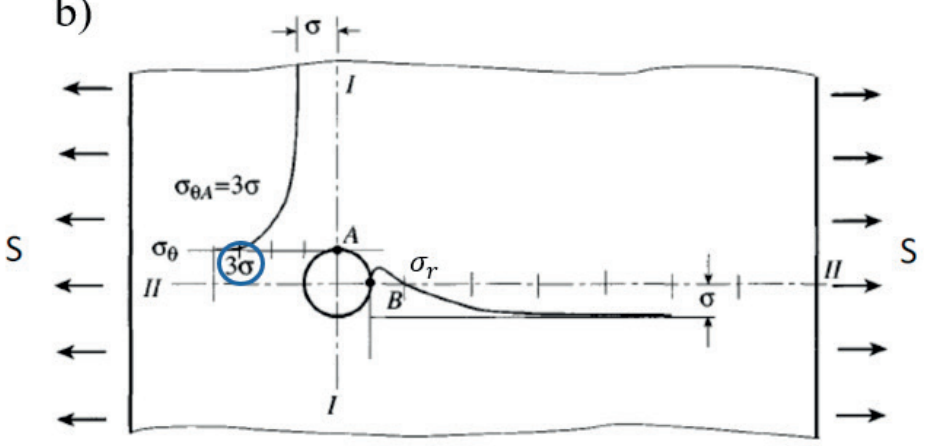

Figure 1 Analytical model (a) and stress distribution (b) for a uniaxially loaded infinite holed plate

a)

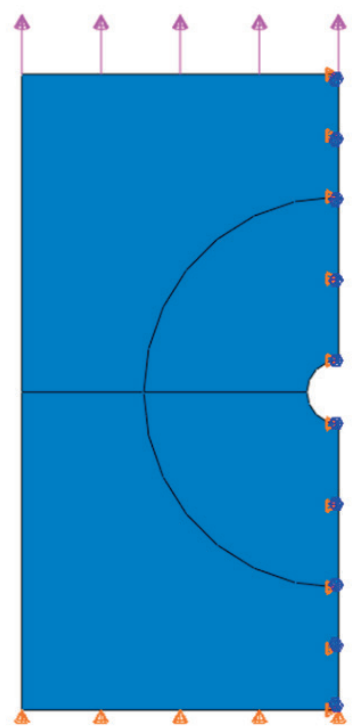

b)

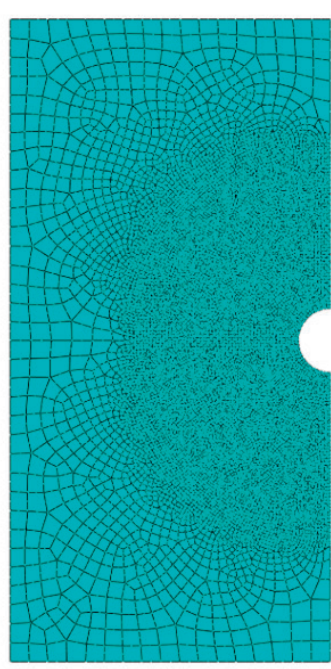

c)

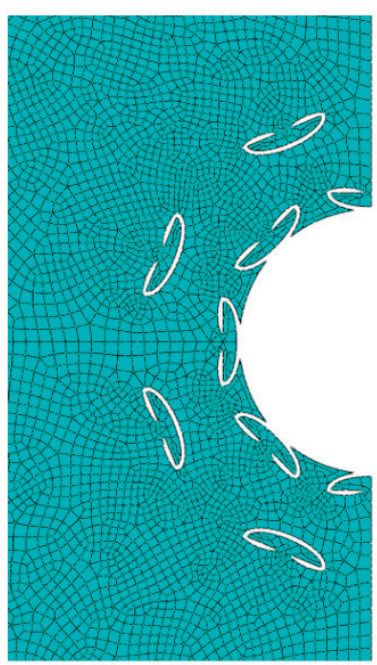

Figure 2 Numerical model

\section{Stress concentration around a circular hole}

The case of an infinite plate with a circular hole of radius $\mathrm{R}$ and subjected to a remote uniaxial tensile stress $\mathrm{S}$, Figure 1a, is considered. The analytical formulation of the stress distribution is given by Airy's stress functions. For axisymmetric problems, such formulas can be conveniently represented in cylindrical coordinates, because of their dependence on the radial coordinate only, and presented in Equation 1.

$$
\left\{\begin{array}{c}
\sigma_{r}=\frac{1}{2} S\left(1-\frac{a^{2}}{r^{2}}\right)+\frac{1}{2} S\left(1-\frac{4 a^{2}}{r^{2}}+\frac{3 a^{4}}{r^{4}}\right) \cos (2 \theta) \\
\sigma_{\theta}=\frac{1}{2} S\left(1-\frac{a^{2}}{r^{2}}\right)-\frac{1}{2} S\left(1+\frac{3 a^{4}}{r^{4}}\right) \cos (2 \theta) \\
\tau_{r \theta}=-\frac{1}{2} S\left(1+\frac{2 a^{2}}{r^{2}}-\frac{3 a^{4}}{r^{4}}\right) \sin (2 \theta)
\end{array}\right.
$$

In Figure $1 \mathrm{~b}$ the stress field described by Airy's stress functions is presented. The most critical point is placed perpendicular to the loading direction, for which at the hole boundary the stress value increases by a factor of 3 . Clearly this is the crucial issue related to fatigue strength and justifies the efforts devoted to reducing the notch effect. A method based on the introduction of appropriate void patterns is presented and the results are critically discussed in view of a possible more general application to mechanical components for mitigating the present stress concentration. This kind of void patterns could be easily realized employing the laser cutting technology.

\section{Feature design}

As remarked in previous section, the case of an infinite holed plate is well known in literature and an analytical formulation is given by the Airy's stress function. However, the introduction of additional geometrical discontinuities makes difficult to obtain a simple and valid analytical formulation as well.

For this reason, a numerical model has been built to estimate the stress field for a holed infinite plate subjected to uniaxial load, Figure 2. The ABAQUS CAE software is employed for all numerical analysis. The plane stress 2D elements CPS4 type were used for discretizing the model (Figure 2b), whereas in Figure 2c a magnified view of the meshed region in proximity of the hole boundary is presented.

In particular, the mesh dimension goes from $3 \mathrm{~mm}$ (in the region far from the hole) to $0.5 \mathrm{~mm}$ (in the region interested by the hole and pattern). It is important to highlight that the mesh underwent a convergence analysis by changing the 
a)
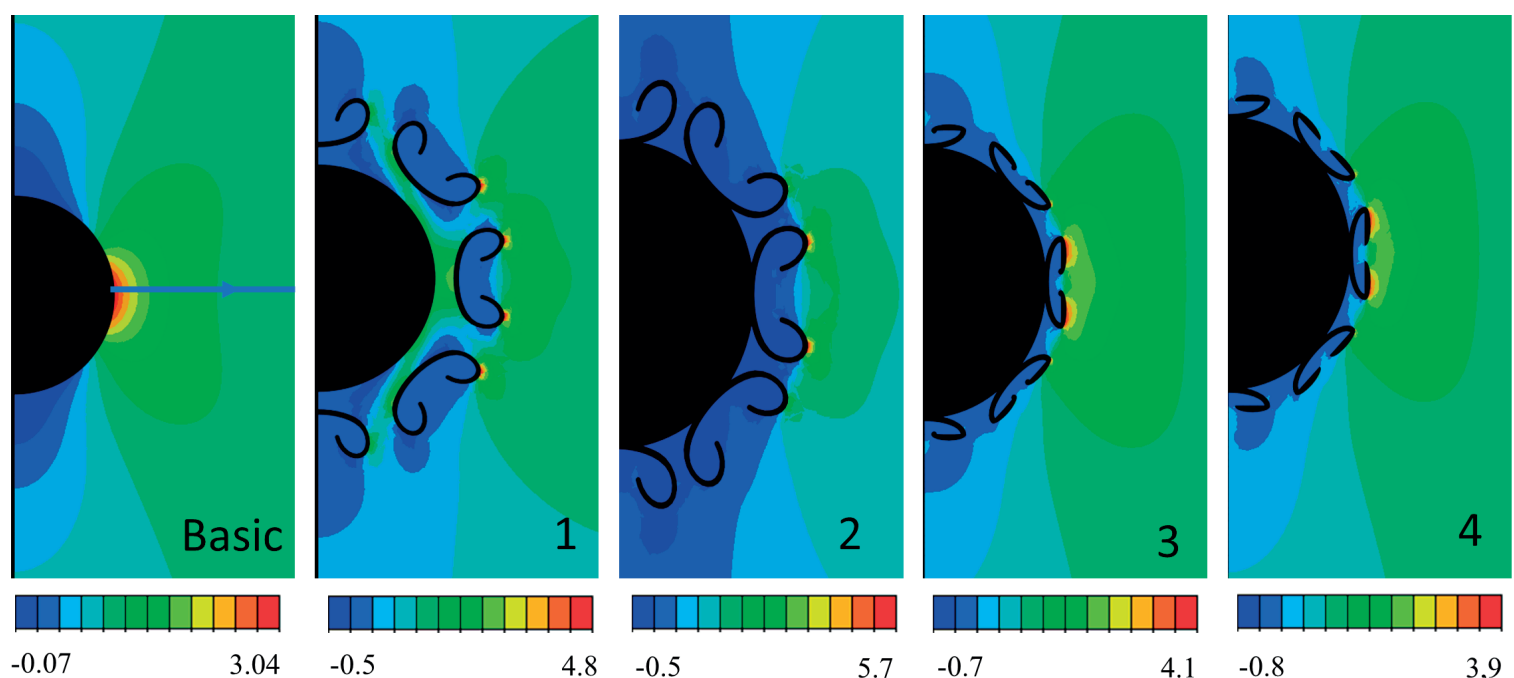

$-0.5$

$-0.5$

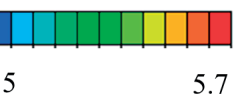

$-0.7$

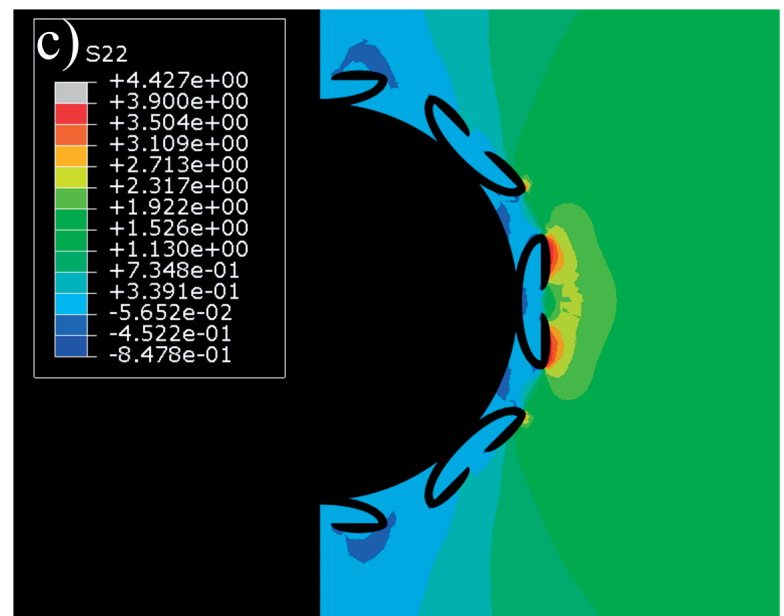

Figure 3 (a) Feature shape evolution; (b) Stress distribution along the "critical direction"; (c) particular view of the stress field near the chosen feature shape. (SCF means "Stress Concentration Factor")

finest mesh dimension from 2 to $0.1 \mathrm{~mm}$. The $0.5 \mathrm{~mm}$ value is chosen as a compromise between the computational effort and being in a surely converged condition. Moreover, the material considered is a generic stainless steel having Young Modulus $E=193 \mathrm{GPa}$ and Poisson's ratio $v=0.33$, as used in [15]. To reduce the computational effort, a symmetric boundary condition has been applied. The model is subjected to a remote stress with magnitude of $S=1 \mathrm{MPa}$; in such a way the results can be considered directly as Stress Concentration Factor values.

As previously anticipated, a C-shaped void pattern is introduced with the aim of redistributing the stress field around the hole in an infinite plate. Grima et al. [13] claim that the S-shaped void induces a soft mode of deformation in the surrounding material based on rotation of the material around. However, it is considered valuable to exploit the polar distribution, instead of the Cartesian. Clearly, a C-shaped element has a symmetry axis that makes it more valuable for the polar distribution than the S-shaped one (antisymmetric). The definition of an optimized pattern passes through a series of design steps in which the feature shape, as well as its position relatively to the hole is modified with the aim of reducing the stress concentration factor along the critical direction, that is the one perpendicular to the load direction. Clearly, once a suitable feature-to-hole relative position has been defined, the feature design improvements objective consists of a compromise between the minimization of the stress along the critical direction and the induced stress concentration at the feature extremes. In Figure 3a, the feature shape evolution series is shown, as well as the stress distribution along the direction of interests in Figure $3 \mathrm{~b}$. In Figure 3c, a magnified view of the stress field around the final feature design is presented.

\section{Ligament length}

It is well known that introducing geometrical discontinuities in mechanical components generates stress concentration effects that are detrimental for the mechanical performances. The adoption of the C-Shape void pattern showed, in the previous section, the possibility of managing the stress distribution, meaning that a compromise must be reached. The modification of stress field clearly moves the potential crack initiation point from the hole boundary to the feature tip. Furthermore, considering that numerical analysis shows 

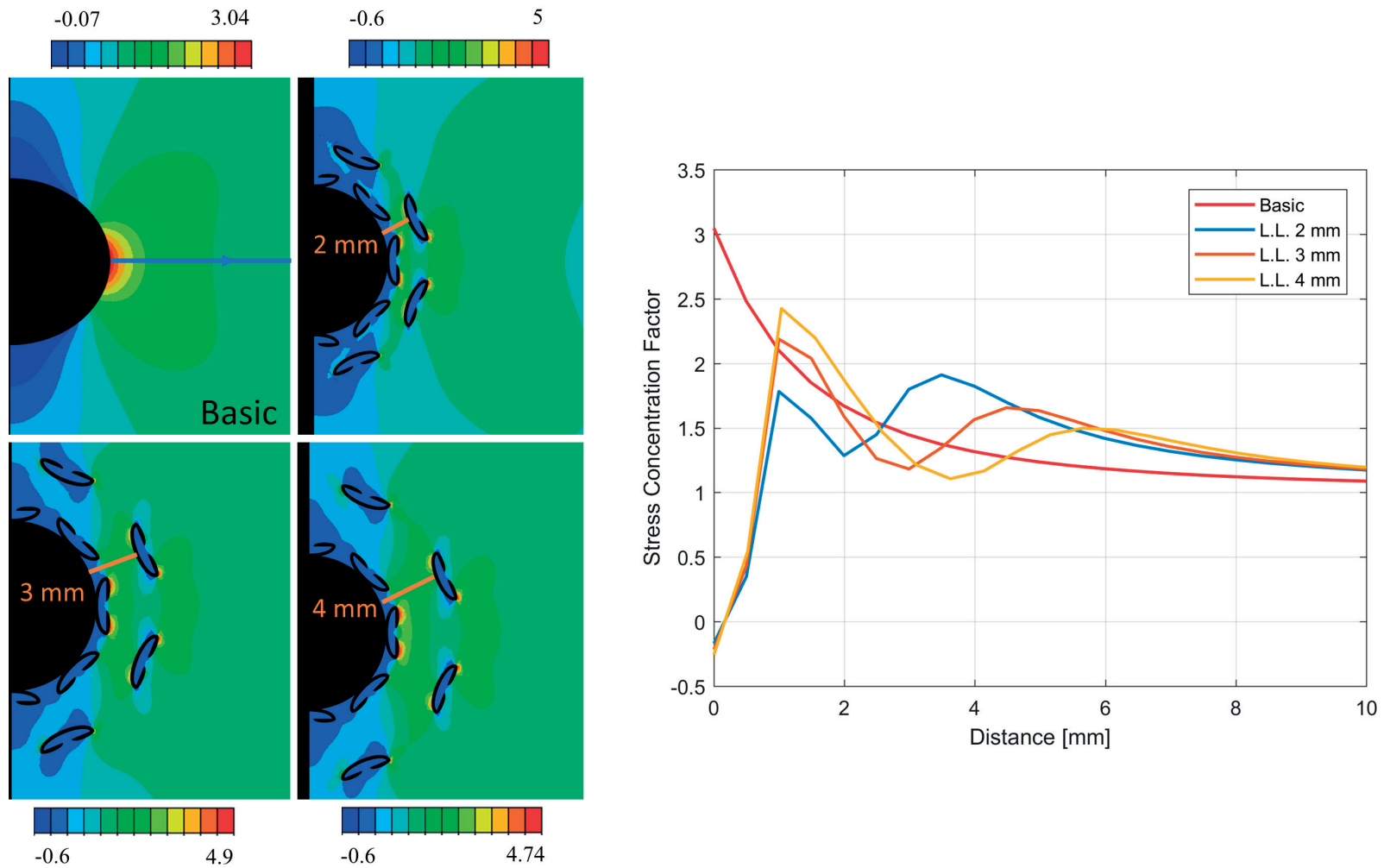

Figure 4 Stress distribution along the "critical direction" for two-row-pattern case (considering the S22 as shown in Figure 3c)
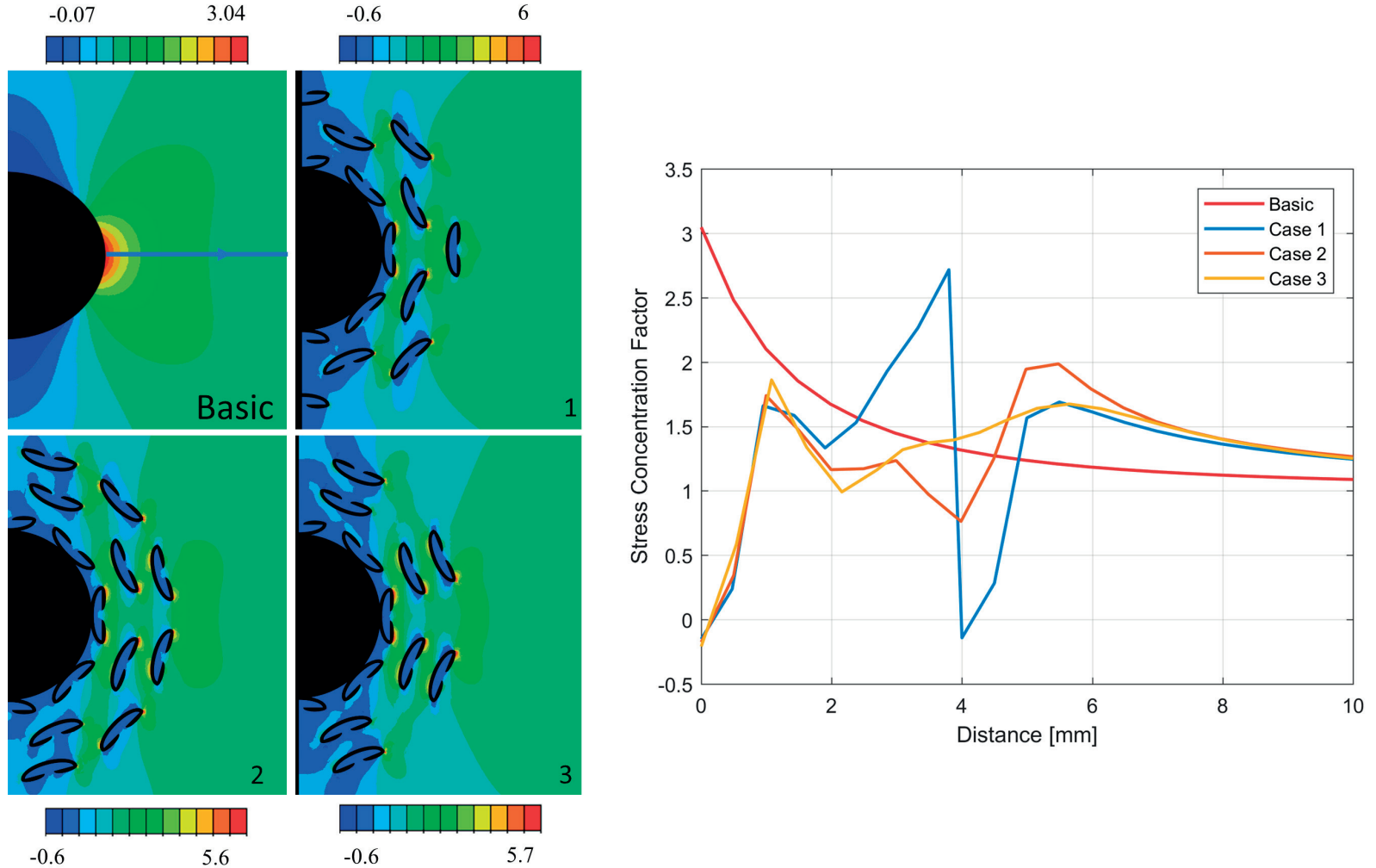

Figure 5 Stress distribution along the "critical direction" for three-row pattern case (considering the S22 as shown in Figure 3c)

the stress concentration values slightly higher than for the case of a simple holed plate, the presented solution can be more susceptible to crack fatigue damage. Clearly, to manage the crack propagation phenomena it is not possible to adopt a single row pattern. In [15] the so-called Ligament Length is used for representing the minimum distance between two contiguous pattern features. Even in the present case, it is interesting to determine the Ligament Length, defined as a distance between the two contiguous rows, whose value helps to reduce the stress concentration factor along the 


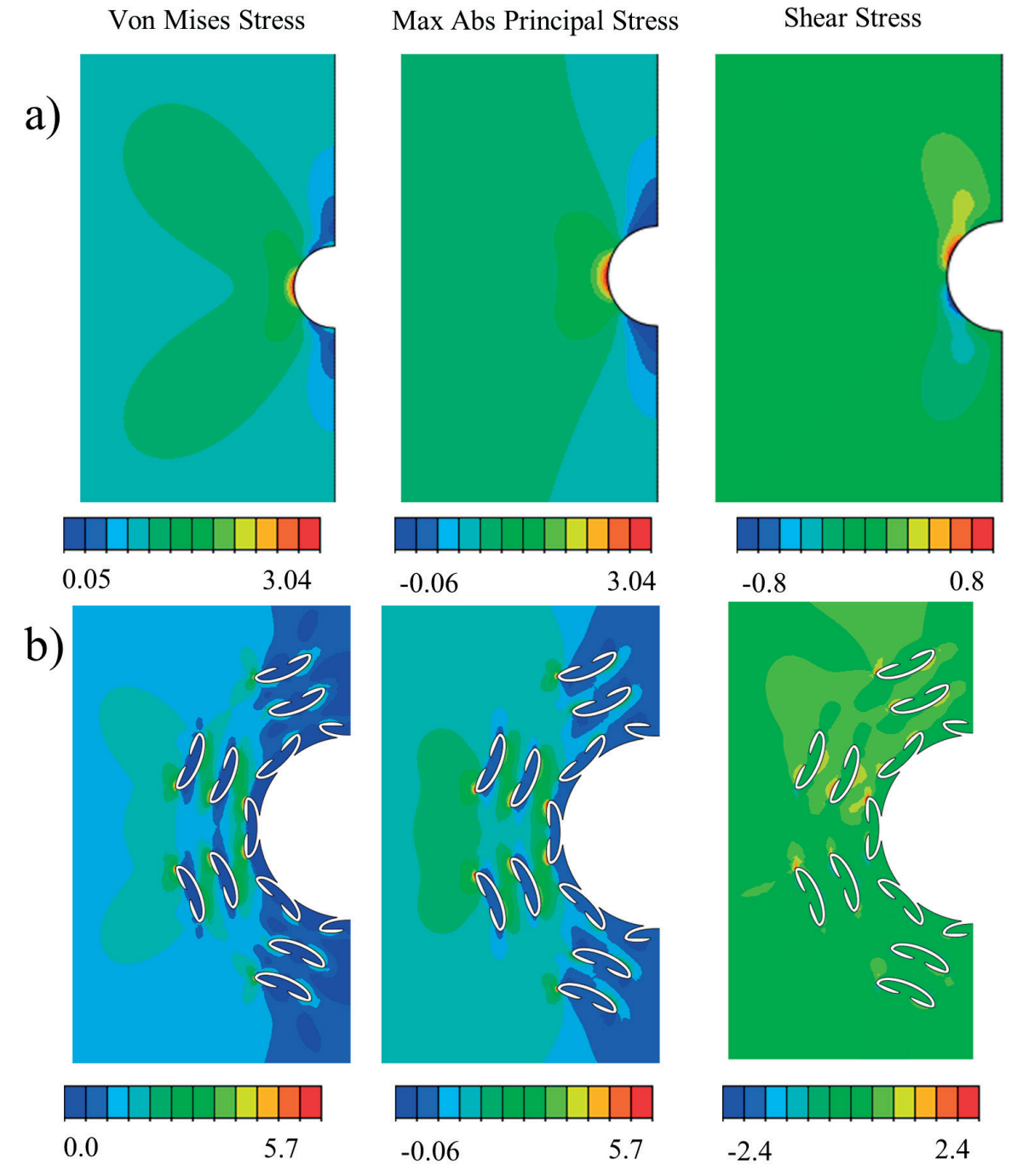

Figure 6 Results in terms of Von Mises Stress, Max. Abs. Principal Stress and Shear Stress for: (a) the basic case; (b) the chosen pattern

critical direction. Three cases have been considered adopting a Ligament Length of $2 \mathrm{~mm}, 3 \mathrm{~mm}$ and $4 \mathrm{~mm}$, as shown in Figure 4. The resulting stress profiles shows that the most convenient Ligament Length is $2 \mathrm{~mm}$ since this solution provides the more effective stress concentration reduction.

\section{Pattern improvement}

Considering the stress profile obtained in the case of a single row pattern, comparison to one obtained with two-row pattern, it appears that the introduction of additional pattern rows contributes the redistribution of the stress field and reduce the maximum stress value reached. For this reason, a third row is added even adopting a Ligament Length of $2 \mathrm{~mm}$. Looking at Figure 5, immediately appears that the resulting stress profile is affected by the features position, as already assessed in Section 3. For this reason, the three different cases, shown in Figure 5, are studied. The most effective stress reduction is reached adopting the case number 3, with a final Stress Concentration Factor reduction of nearly $38 \%$ with respect to the initial value.

\section{Evaluation of the whole model for the chosen solution}

Despite the results obtained relatively to the critical direction, it is clear that the introduction of geometrical discontinuities introduces regions of high stress concentration requiring an evaluation with respect to the basic case of a single hole. In order to make this comparison, the Von Mises Stress, the Maximum Principal Stress, as well as the Shear Stress are evaluated for the chosen solution (presented in Figure $6 \mathrm{a}$ for the basic case, Figure $6 \mathrm{~b}$ for the chosen pattern). A great difference in the stress distribution can be observed, as well as different areas of the stress concentration. Such stress concentration area is very small at the extremes of the single pattern feature, leading to a strong stress gradient. Despite the value itself of the stress concentration introduced by the void pattern, for the scope of this work the effort was focused to understanding the influence of the void pattern relatively to the stress along the critical direction. Consequently, the introduced C-shaped feature must be optimized in order to reduce the induced stress concentration at its extremes as much as possible. 


\section{Conclusion}

Despite the several studies found in literature about introducing the auxiliary holes to provide a global stress concentration factor reduction by redistributing the load as an equivalent ellipse, the present study successfully introduced the C-shaped void patter to manage the stress field and reaching a reduction of the stress concentration factor of about $38 \%$. Clearly, this result is concerned with the "critical direction" that is well known in literature as the one perpendicular to the loading direction. However, the introduction of the strange shaped discontinuities generates stress concentration effects at the tips of these features, leading to a stress concentration increment of about 1.8. Simultaneously, unlike in the basic case, in the chosen pattern the stress gradient is very high, meaning that the stress increment is limited to a small area. The potential of introducing a structured pattern instead of a single feature stands in the possibility of "controlling" the crack propagation phenomenon, or at least to provide a method to detect the propagating crack before that reaches the critical value.

The present study introduces several research possibilities, in particular it is interesting to investigate whether the fatigue resistance is improved by introduction of the porous structure, as well as the application of such a solution to a defined case study. In addition, both feature design, as well as the pattern definition must be optimized for reaching the two main objectives: reducing the induced stress concentration factor at the feature tip and making the performance improvement independent of the load case.

\section{Acknowledgements}

The author wants to thank prof. Mario Guagliano for his support during the development of this work.

\section{References}

[1] DHIR, S. K.: Optimization in a Class of Hole Shapes in Plate Structures. Journal of Applied Mechanics, 48(4), 905-908, 1981.

[2] WU, Z:: Optimal Hole Shape for Minimum Stress Concentration Using Parameterized Geometry Models. Structural and Multidisciplinary Optimization, 37(6), 625-634, 2009. https://doi.org/10.1007/s00158-008-0253-4

[3] YOUNIS, N.T.: Assembly Stress for the Reduction of Stress Concentration. Mechanics Research Communications, 33(3), 837-845, 2006.

[4] WU, H.-C., MU, B.: On Stress Concentrations for Isotropic/Orthotropic Plates and Cylinders with a Circular Hole. Composites Part B: Engineering, 34(2), 127-134, 2003.

[5] KO, W. L.: Stress Concentration around a Small Circular Hole in the HiMAT Composite Plate. NASA Technical Memorandum $86038,1-15,1985$.

[6] TOUBAL, L., KARAMA, M., LORRAIN, B.: Stress Concentration in a Circular Hole in Composite Plate. Composite Structures, 68(1), 31-36, 2005. https://doi.org/10.1016/j.compstruct.2004.02.016

[7] DAN-JUMBO, E., KELLER, R., CHAN, W. S., SELVARAJ, S.: Strength of Composite Laminate with Multiple Holes. Proceedings of 17th International conference on composite materials (CCM-17), United Kingdom, 27-31, 2009.

[8] MEGUID, S. A.: Finite Element Analysis of Defence Hole Systems for the Reduction of Stress Concentration in a UniaxiallyLoaded Plate with Two Coaxial Holes. Engineering Fracture Mechanics, 25(4), 403-413, 1986. https://doi.org/10.1016/00137944(86)90254-7

[9] AKOUR, S.N., AL-HUSBAN, M., NAYFEH, J.F.: Design and Optimization of Defense Hole System for Hybrid Loaded Laminates. Technology Engineering and Management in Aviation: Advancements and Discoveries, IGI Global, 151-160, 2012.

[10] JAIN, D. N.: The Reduction of Stress Concentration in a Uni-Axially Loaded Infinite Width Rectangular Isotropic/Orthotropic Plate with Central Circular Hole by Coaxial Auxiliary Holes. IIUM Engineering Journal, 12(6), 2012.

[11] JINDAL, U. C.: Reduction of Stress Concentration around a Hole in a Uniaxially Loaded Plate. The Journal of Strain Analysis for Engineering Design, 18(2), 135-141, 1983.

[12] PROVIDAKIS, C. P., SOTIROPOUlOS, D. A., BESKOS, D. E.: BEM Analysis of Reduced Dynamic Stress Concentration by Multiple Holes. International Journal for Numerical Methods in Biomedical Engineering, 9(11), 917-924, 1993. https://doi. org/10.1002/cnm.1640091108

[13] GRIMA, J. N., GATT, R.: Perforated Sheets Exhibiting Negative Poisson’s Ratios. Advanced Engineering Materials, 460-464, 2010. https://doi.org/10.1002/adem.201000005

[14] TAYLOR, M., FRANCESCONI, L., GERENDAS, M., SHANIAN, A., CARSON, C., BERTOLDI, K.: Low Porosity Metallic Periodic Structures with Negative Poisson's Ratio. Advanced Materials, 26(15), 2365-2370, 2014. https://doi.org/10.1002/ adma.201304464

[15] JAVID, F., LIU, J., RAFSANJANI, A., SCHAENZER, M., PHAM, M. Q., BACKMAN, D., YANDT, S., INNES, M. C., BOOTHMORRISON, CH., GERENDAS, M., SCARINCI, T., SHANIAN, A., BERTOLDI, K.: On the Design of Porous Structures with Enhanced Fatigue Life. Extreme Mechanics Letters, 13-17, 2017. https://doi.org/10.1016/j.eml.2017.08.002 